\title{
Ingestibilité et digestibilité de deux fourrages tropicaux distribués en l'état, traités à l'urée ou complémentés en matières azotées
}

\author{
AJ Nianogo ${ }^{1}$, V Bougouma-Yaméogo ${ }^{1 *}$, R Cordesse $^{2}$ \\ 'Département de zootechnie et de biologie animale, Inera, Kamboinsé, \\ BP 7192, Ouagadougou, Burkina Faso; \\ ${ }^{2}$ Unité de zootechnie méditerranéenne, Ensa.M-Inra Montpellier, place Pierre Viala, \\ 34060 Montpellier cedex 1, France
}

(Reçu le 27 novembre 1996 ; accepté le 10 juin 1997)

\begin{abstract}
Summary - Intake and digestibility of two tropical grasses fed untreated, urea-treated or supplemented with nitrogen. Intake and in vivo digestibility of Pennisetum pedicellatum Trin ( $\mathrm{Pp}$, trial 1) and of Scheonefeldia gracilis (Sg, trials 2 and 3) were compared. The grasses were harvested either at boot stage and sun-cured, or late at seed dissemination stage, and distributed in one of three modes: untreated, after treatment with $6 \%$ urea, or supplemented with nitrogen in amounts equivalent to those of the urea treatment. Amounts refused by the animals were $19 \pm 4 \%, 21 \pm 4 \%$ and $40 \%$, respectively in trials 1,2 and 3 . Urea treatment improved feed intake from $41.1 \mathrm{~g}$ per $\mathrm{kg}$ metabolic body weight (kg MBW) for untreated $\mathrm{Sg}$ to $67.2 \mathrm{~g} / \mathrm{kg} \mathrm{MBW}$ for treated $\mathrm{Sg}$; it also improved chemical component digestibility and increased the nutritive value of treated forage to the level of early harvested forage (tables III, IV). The presence of inflorescences limits the intake of Sg harvested early $(34.7 \mathrm{~g} / \mathrm{kg} \mathrm{MBW})$ and the $2 \%$ urea treatment restores it $(52.5 \mathrm{~g} / \mathrm{kg} \mathrm{MBW})($ table $\mathrm{V})$. The increase in straw dry matter intake and in digestibility of straw organic matter following urea treatment may be explained by the chemical changes related to the breaking of the lignin-polysaccharide links wich favors the release of digestible components. The supplementation of late harvested forage with cottonseed meal increases the intake and digestibility of organic matter (tables III, IV). This may be due to greater stimulation of ruminal flora as a result of increased availability of energy and nitrogen-providing nutrients. With $\mathrm{Pp}$, supplementation is more efficient than chemical treatment, in contrast with Sg. This is probably linked with differing charasteristics between the two forages. Results indicate that the utilisation of tropical grass straws by sheep may be significantly improved by urea
\end{abstract}

\footnotetext{
* Correspondance et tirés à part
}

Tél : (226) 319229 ; fax : (226) 319206 ou 340271 ; e-mail : panimale@ fasonet.bf 
treatment, or by supplementation. The decision of a farmer with regard to which technique is more appropriate will depend on the grass species, and on the specific socio-economic environment.

\title{
Pennisetum pedicellatum Trin and Schoenefeldia gracilis / urea treatment / supplementation / intake / digestibility / sheep
}

\begin{abstract}
Résumé - L'ingestibilité et la digestibilité in vivo de Pennisetum pedicellatum Trin (Pp) et Schoenefeldia gracilis $(\mathrm{Sg})$ ont été comparées. Les fourrages ont été récoltés soit précocement et conservés sous forme de foin, soit tardivement et distribués en l'état ou traités à l'urée ou encore complémentés en matières azotées en quantité équivalente à celle apportée par le traitement à l'urée. Le traitement à $6 \%$ d'urée après humidification à $40 \%$ améliore les quantités ingérées $\left(67,2 \mathrm{~g} / \mathrm{kgP}^{0,75}\right.$ pour $\mathrm{Sg}$ traité vs $41,1 \mathrm{~g} / \mathrm{kgP}^{0,75}$ sans traitement), la digestibilité des composants chimiques, et amène la valeur nutritive du fourrage traité au niveau de celui de la récolte précoce. La complémentation du fourrage récolté tardivement avec du tourteau de coton améliore l'ingestibilité et la digestibilité de la matière organique. La présence des inflorescences limite l'ingestibilité de Sg récolté précocement et le traitement à $2 \%$ d'urée la restaure : $52,5 \mathrm{~g} / \mathrm{kgP}^{0,75}$ contre 34,7 avec le fourrage non traité.
\end{abstract}

Pennisetum pedicellatum Trin / Schoenefeldia gracilis / traitement à l'urée / complémentation / ingestibilité / digestibilité / moutons

\section{INTRODUCTION}

Dans les pays sahéliens, l'alimentation du cheptel est basée essentiellement sur le pâturage naturel, sujet à d'énormes fluctuations tant quantitatives que qualitatives. L'avenir de l'élevage dans ces pays dépend d'une meilleure valorisation des ressources végétales spontanées. L'exploitation précoce des fourrages permet d'assurer les besoins d'entretien des animaux et une faible production (Zoungrana, 1994). En revanche, leur exploitation tardive, après fructification, permet rarement de couvrir les besoins d'entretien (Guerin et al, 1990).

Les études réalisées in vitro sur quatre fourrages tropicaux ont montré une modification importante de leur composition chimique et de leur digestibilité avec l'âge (Bougouma-Yaméogo, 1995). En effet, les fourrages tropicaux, malgré leur structure plus lignifiée caractéristique des plantes de type $\mathrm{C} 4$, se comportent comme des pailles cultivées en milieu tempéré (type C3). Des doses de $9 \%$ d'urée provoquent des modifications chimiques aussi importantes que celles entraînées par le traitement à 4,5\% d'ammoniac, dose habituellement utilisée pour le traitement des pailles en milieu tempéré. Malheureusement, malgré une durée de traitement de $30 \mathrm{j}$, des quantités relativement importantes d'urée résiduelle ont été mesurées. Ces quantités peuvent entraîner des risques d'intoxication chez les animaux. Pour ces raisons nous avons adopté une dose de $6 \%$ d'urée, une teneur en humidité de $40 \%$ et une durée de traitement de $30 \mathrm{j}$ (Bougouma-Yaméogo et al, 1996). Ces conditions peuvent-elles à l'échelle de l'exploitation entraîner une amélioration significative de la valeur nutritive des fourrages tropicaux?

Nous nous proposons, dans cet article, de présenter une étude comparative de l'ingestibilité et de la digestibilité de deux fourrages spontanés tropicaux, Pennisetum pedicellatum $(\mathrm{Pp})$ et Schoenefeldia gracilis $(\mathrm{Sg})$, récoltés précocement au stade début épiaison ou tardivement après fructification et dissémination des graines. Les fourrages récoltés à l'état pailleux sont distribués en l'état ou complémentés en matières azotées par du tourteau de coton, ou bien après traitement à l'ammoniac généré par uréolyse. Un traitement de conservation à $2 \%$ d'urée 
des fourrages récoltés précocement est également effectué.

\section{MATÉRIELS ET MÉTHODES}

\section{Fourrages}

Pour chacune des espèces, Pennisetum pedicellatum Trin et Schoenefeldia gracilis, il y a eu deux périodes de récolte :

- une récolte précoce, effectuée courant septembre au stade début épiaison, sous forme de foin séché au sol. À ce stade, les fourrages récoltés sont composés essentiellement de feuilles vertes et de feuilles sèches, de quelques tiges avec les inflorescences formées pour Pennisetum pedicellatum Trin, et d'épis pour Schoenefeldia gracilis, pour lequel le stade de début épiaison était en fait déjà dépassé. Sur ce fourrage récolté précocement $(\mathrm{Sg})$ un traitement de conservation à la dose de $2 \%$ d'urée et $40 \%$ d'humidité a été effectué ;

- une récolte tardive au stade dissémination des graines des fourrages très lignifiés, à maturité complète, courant octobre-novembre. Ces fourrages, réduits en brins de $20 \mathrm{à} 30 \mathrm{~cm}$ de long, ont été traités à la dose de $6 \%$ d'urée après humidification à $40 \%$ (Bougouma-Yaméogo, 1995; Chenost et al, 1993). La technique de traitement en meule retenue a été celle décrite par Schiere et al (1988). Pour cela, chaque lit de la meule confectionnée avec des bottes de paille de 20 à $25 \mathrm{~kg}$ de MS a été arrosé avec la quantité nécessaire du mélange eau-urée puis piétiné afin de bien tasser la meule qui a ensuite été recouverte d'un film plastique. Une partie des fourrages n'ayant pas subi le traitement chimique a été mise de côté pour être utilisée en l'état. Après $30 \mathrm{j}$ de traitement, la meule ( 0,5 à $0,8 \mathrm{~m}$ de haut) a été ouverte, et six échantillons destinés au dosage de l'urée résiduelle ont été immédiatement prélevés à trois niveaux (deux par niveau) : au centre et à $20 \mathrm{~cm}$ du haut et du bas. Le reste du produit a été séché au sol et stocké à l’abri.

\section{Animaux}

Les animaux expérimentaux, des moutons, ont subi, pendant la période d'adaptation, un traitement de déparasitage interne et externe, une vaccination contre la pasteurellose et la peste des petits ruminants et ont reçu deux doses d'un complexe vitaminique (vitamines $A$ et $E$ ) à 2 semaines d'intervalle. Nous avons utilisé dans les trois essais un dispositif randomisé.

Pour les mesures de digestibilité (essais 1 et 2), 24 ovins mâles entiers de race Djallonké, de la souche locale 'Mossi', ont été répartis en quatre lots de six animaux. Ils étaient âgés d'environ 15 mois et leurs poids variaient entre 17 et $25 \mathrm{~kg}$. Les animaux, munis de sacs à fèces, ont été maintenus dans des cages à métabolisme permettant de mesurer les quantités d'aliments ingérées et de récupérer les fèces émises chaque jour.

Pour les seules mesures d'ingestibilité (essai 3), nous avons utilisé 20 agnelles de la même race, réparties en deux lots de dix. Elles étaient âgées d'environ 16 mois et leurs poids variaient entre 12 et $18 \mathrm{~kg}$.

\section{Protocole expérimental}

\section{Rations}

La période d'adaptation au régime a été de 3 semaines et a permis de limiter les refus à $20 \%$ des quantités distribuées dans les essais 1 et 2 , et à $40 \%$ dans l'essai 3 . La période de collecte des fèces a été de $10 \mathrm{j}$ dans les essais 1 et 2 , et de 3 semaines dans l'essai d'ingestibilité (essai 3). Les deux fourrages, Pennisetum pedicellatum (essai 1) et Schoenefeldia gracilis (essai 2 et 3), ont été présentés aux animaux de la façon suivante :

\section{Essais 1 et 2}

- Lot 1 : les fourrages récoltés précocement non traités.

- Lot 2 : les fourrages récoltés tardivement non traités. Nous avons cependant dû interrompre, pour cause de sous-alimentation grave, le lot de l'essai 1 alimenté avec $\mathrm{Pp}$.

- Lot 3 : les fourrages récoltés tardivement et traités à $6 \%$ d'urée et $40 \%$ d'humidité.

- Lot 4 : les fourrages récoltés tardivement non traités, complémentés avec du tourteau de coton afin d'amener la teneur en matières azotées des rations au niveau de celle du fourrage traité. 


\section{Essai 3}

Il a permis de mesurer en plus les quantités ingérées de Schoenefeldia gracilis récolté précocement, soit non traité (lot 1 ) soit traité à $2 \%$ d'urée et $40 \%$ d'humidité (lot 2 ) lorsqu' on autorise un niveau élevé de refus.

Dans les trois essais, les fourrages étaient distribués en deux repas, à 9 et $15 \mathrm{~h}$, et le concentré offert en un seul repas avant la distribution du foin du premier repas. L'eau et un complément minéral riche en zinc ( $12 \mathrm{P}$, marque déposée produite par Les Salins du Midi, France) étaient offerts ad libitum tout au long des essais. Les caractéristiques du tourteau de coton étaient de $45,1 \%$ de MAT, $8 \%$ de cellulose brute, $10,7 \%$ de matières grasses, $6,7 \%$ de matières minérales dans la matière sèche. Sa valeur énergétique calculée est de 1,14 UFV $/ \mathrm{kg}$ de MS et 1,17 UFL/kg de MS (Sauvant, 1981).

\section{Collecte des données}

Les quantités de fourrage distribuées et refusées et les quantités de fèces émises ont été mesurées quotidiennement (à $\pm 10 \mathrm{~g}$ de précision) à $9 \mathrm{~h}$. Les refus et les fêces (séchés dans une étuve ventilée réglée à la température de $60^{\circ} \mathrm{C}$ ) ont été regroupés par animal pour la période de récolte. À la fin de cette période, un échantillon a été prélevé après homogénéisation, sur chacun des produits, puis broyé et conservé avant analyses.

\section{Analyses chimiques}

L'urée résiduelle a été dosée sur tous les fourrages traités selon la méthode enzymatique du kit Boerhinger Mannhein $n^{\circ} 542946$.

Les échantillons d'aliments distribués et refusés et de fèces ont fait l'objet des analyses suivantes : teneur en matière sèche (MS), matière organique (MO) et matières azotées totales (MAT) selon les méthodes de l'AOAC (1984) ainsi que les teneurs en Free NDF (neutral detergent fiber déminéralisé), ADF (acid detergent fiber), et ADL (acid detergent lignin) selon les méthodes décrites par Robertson et Van Soest (1981).

\section{Calculs et analyses statistiques}

Les digestibilités ont été calculées pour chaque ration, par animal pour la période de $10 \mathrm{j}$ de col- lecte et pour les constituants chimiques MS, MO, MAT, NDF et ADF. Une analyse de variance a été effectuée en utilisant la procédure GLM (general linear models procedure) de Sas (1982); les moyennes ont été hiérarchisées grâce au test de Scheffe.

\section{RÉSULTATS}

Dans les essais 1 et 2, les pourcentages de refus ont été respectivement de $19 \pm 4 \%$ et $21 \pm 4 \%$. Dans l'essai 3 , le taux de refus a été maintenu volontairement élevé (40\% pour le lot $1,30 \%$ pour le lot 2 ), ce qui nous a amené à distribuer une quantité plus importante de fourrage, soit $4 \%$ et $3,5 \%$ du poids vif, respectivement pour les lots 1 et 2 .

\section{Composition chimique des rations étudiées}

L'urée résiduelle des produits traités a été en moyenne de $3,1 \pm 2,4 \mathrm{mg}$ d'urée/gMS dans les échantillons de Pennisetum pedicellatum Trin. Cette teneur en urée résiduelle n'a pas été prise en compte lors de la complémentation, car l'uréolyse se poursuit après le traitement pendant le séchage du produit traité au soleil (Hassoun, 1987). Si l'urée résiduelle n'était pas résorbée au cours du séchage, elle fournirait au maximum $(3,5+2,6) \times 1,47=7,9 \mathrm{~g}$ de PDIN/kg de MS, valeur ne pouvant provoquer de risques d'intoxication pour les animaux.

La composition chimique des aliments distribués (tableau I) montre que les fourrages non traités récoltés précocement ont une valeur nutritive plus élevée que ceux récoltés tardivement. Vingt-quatre et 13,4\% de tourteau de coton ont été ajoutés aux fourrages non traités récoltés tardivement, respectivement pour $\mathrm{Pp}$ et $\mathrm{Sg}$, afin d'obtenir des rations de teneurs en azote équivalentes à celles des fourrages traités. Le traitement de conservation à $2 \%$ d'urée de Schoene- 
feldia gracilis récolté précocement (essai 3) a augmenté sa teneur en MAT de 3,5 points et n'a pas modifié significativement sa teneur en NDF.

\section{Ingestibilité et digestibilité}

La composition chimique des refus montre que les animaux ont exercé un tri très sélectif. Ils prélèvent les fractions les plus riches en MAT et écartent les fractions les plus lignifiées ainsi que les inflorescences de $\mathrm{Sg}$ (tableaux I et II). Le tourteau est entièrement et rapidement ingéré.

Dans l'essai 1, le fourrage Pennisetum pedicellatum Trin, récolté tardivement non traité (lot 2), n'a pas pu être étudié en raison de l'apparition chez les animaux de signes graves de sous-alimentation. En revanche, les trois autres modes de distribution : fourrage récolté précocement (lot 1 ) et récolté tardivement traité (lot 3) ou non traité mais complémenté avec $24 \%$ de tourteau de coton (lot 4), ont permis d'assurer la couverture des besoins d'entretien des animaux estimés à $23 \mathrm{~g}$ de MODI/ $/ \mathrm{kP}^{0.75}$ (tableau III). La complémentation a donné le meilleur résultat avec un niveau d'alimentation de 2,1. Les deux autres rations ont eu des niveaux très voisins, de l'ordre de 1,2. La comparaison entre les trois rations montre une quantité ingérée supérieure pour la ration complémentée, une moins bonne digestibilité des matières azotées du fourrage récolté précocement (lot 1) et une faible digestibilité de la matière organique (dMO) pour le fourrage traité. La complémentation

Tableau I. Composition chimique des aliments offerts et des aliments refusés dans les essais 1 et 2.

\begin{tabular}{cccccc}
\hline \multirow{2}{*}{ Nature des fourrages } & \multicolumn{2}{c}{$M A T$} & & \multicolumn{2}{c}{$N D F$} \\
\cline { 2 - 3 } \cline { 5 - 6 } & $\begin{array}{c}\text { Pennisetum } \\
\text { pedicellatum } \\
\text { Trin. }\end{array}$ & $\begin{array}{c}\text { Schoenefeldia } \\
\text { gracilis }\end{array}$ & & $\begin{array}{c}\text { Pennisetum } \\
\text { pedicellatum } \\
\text { Trin }\end{array}$ & $\begin{array}{c}\text { Schoenefeldia } \\
\text { gracilis }\end{array}$ \\
\hline
\end{tabular}

Lot 1

$\begin{array}{llllll}\text { fourrage précoce } & \text { offert } & 6,0 \pm 0,1 & 6,1 \pm 0,2 & 75,0 \pm 0,3 & 73,8 \pm 0,3 \\ \text { non traité } & \text { refusé } & 3,0 \pm 0,2 & 7,5 \pm 0,5 & 84,9 \pm 0,4 & 78,7 \pm 0,7\end{array}$

Lot 2

\begin{tabular}{|c|c|c|c|c|c|}
\hline $\begin{array}{l}\text { fourrage tardif } \\
\text { non traité }\end{array}$ & $\begin{array}{l}\text { offert } \\
\text { refusé }\end{array}$ & $\begin{array}{c}4,0 \pm 0,2 \\
-\end{array}$ & $\begin{array}{l}2,2 \pm 0,1 \\
1,9 \pm 0,4\end{array}$ & $\begin{array}{c}79,8 \pm 0,1 \\
-\end{array}$ & $\begin{array}{l}75,1 \pm 0,3 \\
81,7 \pm 1,2\end{array}$ \\
\hline $\begin{array}{l}\text { Lot } 3 \\
\text { fourrage tardif } \\
\text { traité }\end{array}$ & $\begin{array}{l}\text { offert } \\
\text { refusé }\end{array}$ & $\begin{array}{r}14,4 \pm 0,2 \\
9,8 \pm 0,9\end{array}$ & $\begin{array}{l}9,4 \pm 0,1 \\
7,9 \pm 0,5\end{array}$ & $\begin{array}{l}75,8 \pm 0,4 \\
86,3 \pm 1,1\end{array}$ & $\begin{array}{l}72,4 \pm 0,1 \\
78,7 \pm 1,2\end{array}$ \\
\hline $\begin{array}{l}\text { Lot } 4 \\
\text { fourrage tardif } \\
\text { non traité } \\
+ \text { tourteau de coton } \\
\text { inclus }\end{array}$ & $\begin{array}{l}\text { offert } \\
\text { refusé }\end{array}$ & $\begin{array}{r}15,6 \pm 0,1 \\
3,1 \pm 0,7\end{array}$ & $\begin{array}{l}8,5 \pm 0,3 \\
2,8 \pm 0,6\end{array}$ & $\begin{array}{l}73,2 \pm 0,2 \\
86,1 \pm 1,7\end{array}$ & $\begin{array}{l}73,7 \pm 0,2 \\
82,2 \pm 0,9\end{array}$ \\
\hline
\end{tabular}

Les teneurs sont exprimées en \% de la MS. Les moyennes et les écart types pour les rations offertes.sont calculés à partir de deux valeurs tandis que ceux des refus sont calculés à partir de six valeurs. 
Tableau II. Quantités totales de matières azotées offertes et ingérées (en g MAT par animal et par jour) selon la ration.

\begin{tabular}{lcc}
\hline Nature des fourrages & $\begin{array}{c}\text { Pennisetum } \\
\text { pedicellatum Trin }\end{array}$ & $\begin{array}{c}\text { Schoenefeldia } \\
\text { gracilis }\end{array}$ \\
\hline
\end{tabular}

Lot 1

fourrage précoce non traité

MAT offertes

Lot 2

fourrage tardif non traité

MAT offertes

$\begin{array}{cc}- & 11,0 \\ - & 8,1\end{array}$

Lot 3

fourrage tardif traité

MAT offertes

89,7

72,7

MAT ingérées

76,5

61,0

Lot 4

fourrage tardif non traité

MAT offertes

117,4

52,4

+ tourteau de coton inclus

MAT ingérées

114,5

50,1

Tableau III. Ingestibilité des rations à base de Pennisetum pedicellatum Trin, récolté précocement ou tardivement, traité à l'urée ou complémenté avec du tourteau de coton. Digestibilité des composants chimiques (essai 1).

\begin{tabular}{|c|c|c|c|c|c|}
\hline Variables & $\begin{array}{c}\text { Lot l : Pp } \\
\text { récolté } \\
\text { précocement } \\
\text { non traité }\end{array}$ & $\begin{array}{l}\text { Lot } 3: P p \\
\text { récolté } \\
\text { tardivement } \\
\text { traité }\end{array}$ & $\begin{array}{l}\text { Lot } 4: \text { Pp récolté } \\
\text { tardivement non } \\
\text { traité }+ \text { touteau }\end{array}$ & $\begin{array}{c}\text { Erreur } \\
\text { standard }\end{array}$ & $\operatorname{Pr}>F$ \\
\hline QI (gMS/kg PV) & $24,3^{\mathrm{b}}$ & $25,3^{b}$ & $37,8^{\mathrm{a}}$ & 0,6 & 0,0001 \\
\hline QI (gMS/kg $\left.\mathrm{P}^{0,75}\right)$ & $50,9^{b}$ & $53,4^{\mathrm{b}}$ & $80,9^{\mathrm{a}}$ & 1,4 & 0,0001 \\
\hline $\operatorname{dMS}(\%)$ & $58,3^{\mathrm{ab}}$ & $55,4^{\mathrm{b}}$ & $62,3^{\mathrm{a}}$ & 0,9 & 0,009 \\
\hline $\mathrm{dMO}(\%)$ & $60,0^{\mathrm{ab}}$ & $58,4^{\mathrm{b}}$ & $68,3^{\mathrm{a}}$ & 1,1 & 0,02 \\
\hline $\mathrm{dNDF}(\%)$ & $64,5^{\mathrm{a}}$ & $63,6^{\mathrm{a}}$ & $63,7^{\mathrm{a}}$ & 0,8 & 0,05 \\
\hline dADF $(\%)$ & $66,0^{\mathrm{a}}$ & $66,0^{\mathrm{a}}$ & $61,8^{\mathrm{a}}$ & 0,7 & 0,04 \\
\hline dMAT (\%) & $42,0^{\mathrm{b}}$ & $72,2^{\mathrm{a}}$ & $73,8^{\mathrm{a}}$ & 1,1 & 0,0001 \\
\hline MODI (gMS/kgP $\left.\mathrm{gP}^{0,75}\right)$ & 27,6 & 29,2 & 49,0 & - & - \\
\hline
\end{tabular}

Les lettres $\left({ }^{a}\right.$ et ${ }^{b}$ ), lecture en ligne, lorsqu'elles diffèrent, indiquent une différence significative au seuil $(p<0,05)$. 'Le tourteau représente $24 \%$ de la MS de la ration globale ingérée, soit 220 à 250 g suivant les quantités ingérées par animal. QI = quantité ingérée, MODI = matière organique digestible ingérée. $\mathrm{Pr}=\mathrm{Probabilité}$. F est le rapport de la somme des moyennes des carrés pour les traitements pour chaque lot sur la somme des moyennes des carrés de l'erreur. Toutes les valeurs présentées correspondent à la moyenne de six résultats individuels. 
importante en tourteau influence significativement la dMO de la ration du lot 4.

Les résultats des mesures faites avec le fourrage Schoenefeldia gracilis (tableau IV) sont plus contrastés. Le fourrage récolté tardivement non traité a pu être étudié, cependant la couverture des besoins d'entretien n'a pas été assurée. Le fourrage récolté tardivement traité (lot 3 ) et le même fourrage non traité mais complémenté avec $13,4 \%$ de tourteau de coton (lot 4 ) ont couvert les besoins d'entretien avec des niveaux d'alimentation respectifs de 1,6 et 1,1 . Le traitement à l'urée a amélioré significativement la dMO et la digestibilité du NDF, et de l'ADF (dNDF, dADF) par rapport aux trois autres lots. $\mathrm{Sg}$ récolté précocement non traité (lot 1) présente une faible ingestibilité en raison de la présence des inflorescences qui constituent l'essentiel des quantités refusées. Dans l'essai 3 (tableau V), l'ingestion volontaire apparaît beaucoup plus élevée que dans l'essai 2. Il est possible que cette différence soit due à l'augmentation des quantités de MS offertes. Cependant, seul le lot 2 recevant le fourrage traité avec $2 \%$ d'urée couvre ses besoins d'entretien.

\section{DISCUSSION}

Les résultats obtenus dans les essais 2 et 3 montrent un effet significatif $(p<0,05)$ du traitement à l'urée sur les quantités ingérées et la $\mathrm{dMO}$ (respectivement $+58 \%$ et $+35 \%$ par rapport au fourrage non traité dans l'essai 2). Ces résultats sont conformes à ceux obtenus avec les nombreuses pailles de céréales traitées à l'urée ou à l'ammoniac (Nefzaoui, 1994 ; Nyarko-Badohu et al, 1994), ou avec des fourrages tropicaux (Cynodon nlemfuensis) traités à $4 \%$ d'ammoniac (Brown, 1988). Pour l'essai 2 avec Schoenefeldia gracilis le traitement

Tableau IV. Ingestibilité des rations à base de Schoenefeldia gracilis récolté précocement ou récolté tardivement, traité ou complémenté avec du tourteau de coton. Digestibilité des composants chimiques (essai 2).

\begin{tabular}{lcccccc}
\hline & $\begin{array}{c}\text { Lot 1:Sg } \\
\text { récolté } \\
\text { précocement } \\
\text { non traité }\end{array}$ & $\begin{array}{c}\text { Lot 2:Sg } \\
\text { récolté } \\
\text { tardivement } \\
\text { non traité }\end{array}$ & $\begin{array}{c}\text { Lot 3:Sg } \\
\text { récolté } \\
\text { tardivement } \\
\text { traité }\end{array}$ & $\begin{array}{c}\text { Lot } 4: S g \\
\text { récolté } \\
\text { tardivement } \\
+ \text { tourteaul }\end{array}$ & $\begin{array}{c}\text { Erreur } \\
\text { standard }\end{array}$ & Pr $>F$ \\
\hline QI (gMS/kg PV) & $14,1^{\mathrm{d}}$ & $20,0^{\mathrm{c}}$ & $31,6^{\mathrm{a}}$ & $25,5^{\mathrm{b}}$ & 0,5 & 0,0001 \\
QI (gMS/kg P,75) & $28,1^{\mathrm{d}}$ & $41,4^{\mathrm{c}}$ & $67,2^{\mathrm{a}}$ & $54,1^{\mathrm{b}}$ & 1,1 & 0,0001 \\
dMS (\%) & $45,2^{\mathrm{b}}$ & $40,2^{\mathrm{c}}$ & $55,3^{\mathrm{a}}$ & $46,5^{\mathrm{b}}$ & 0,8 & 0,0001 \\
dMO (\%) & $52,7^{\mathrm{b}}$ & $45,8^{\mathrm{c}}$ & $61,7^{\mathrm{a}}$ & $57,5^{\mathrm{b}}$ & 0,8 & 0,0001 \\
dNDF (\%) & $53,0^{\mathrm{b}}$ & $47,3^{\mathrm{b}}$ & $73,2^{\mathrm{a}}$ & $51,6^{\mathrm{b}}$ & 0,9 & 0,0001 \\
dADF (\%) & $55,3^{\mathrm{ab}}$ & $51,5^{\mathrm{b}}$ & $61,7^{\mathrm{a}}$ & $52,5^{\mathrm{b}}$ & 0,8 & 0,002 \\
dMAT (\%) & $53,3^{\mathrm{a}}$ & $-63,7^{\mathrm{b}}$ & $41,8^{\mathrm{a}}$ & $55,5^{\mathrm{a}}$ & 1,8 & 0,0001 \\
MODI (gMS/kg P & 13,2 & $\left.17,1^{0,75}\right)$ & 37,3 & 24,7 & - & - \\
\hline
\end{tabular}

Les lettres $\left({ }^{a},{ }^{b}\right.$ et $\left.{ }^{c}\right)$, lecture en ligne, lorsqu'elles diffèrent, indiquent une différence significative $(p<0,05)$. ${ }^{1} \mathrm{Le}$ tourteau représente $13,4 \%$ de la MS de la ration totale, soit 62 à $124 \mathrm{~g}$ en fonction des quantités ingérées par animal. QI = quantité ingérée, $\mathrm{MODI}=$ matière organique digestible ingérée. $\mathrm{Pr}=$ Probabilité. F est le rapport de la somme des moyennes des carrés pour les traitements pour chaque lot sur la somme des moyennes des carrés de l'erreur. Toutes les valeurs présentées correspondent à la moyenne de six résultats individuels. 
Tableau V. Ingestibilité de Schoenefeldia gracilis récolte précoce non traitée et traitée à $2 \%$ d'urée (essai 3).

\begin{tabular}{lcccc}
\hline Variables & $\begin{array}{c}\text { Lot 1: Sg récolté } \\
\text { précocement non traité }\end{array}$ & $\begin{array}{c}\text { Lot 2: Sg récolté } \\
\text { précocement traité }\end{array}$ & Erreurstandard & $\operatorname{Pr}>F$ \\
\hline QI (gMS/kg PV) & $18,0^{\mathrm{b}}$ & $27,2^{\mathrm{a}}$ & 0,9 & 0,001 \\
$\mathrm{QI}\left(\mathrm{gMS} / \mathrm{kgP}^{0,75}\right)$ & $34,7^{\mathrm{b}}$ & $52,5^{\mathrm{a}}$ & 1,6 & 0,0006 \\
MODI $\left(\mathrm{gMS} / \mathrm{kgP}^{0,75}\right)$ & 16,4 & 24,6 & - & -
\end{tabular}

Les lettres $\left({ }^{a}\right.$ et $\left.^{b}\right)$, lecture en ligne, lorsqu'elles diffèrent, indiquent une différence significative au seuil $(p<0,05)$. $\mathrm{QI}=$ quantité ingérée, $\mathrm{MODI}=$ matière organique digestible ingérée. $\mathrm{Pr}=$ Probabilité. F est le rapport de la somme des moyennes des carrés pour les traitements pour chaque lot sur la somme des moyennes des carrés de l'erreur. Toutes les valeurs présentées correspondent à la moyenne de dix résultats individuels.

améliore également la dMO et la dNDF (respectivement $+15,9$ et $+25,9$ points). Des résultats similaires ont été obtenus avec de la paille de sorgho traitée à $3 \%$ d'ammoniac ou à $4 \%$ d'urée (Nefzaoui, 1994). Avec un foin tropical (Hemarthria altissima) traité aux doses de 2, 3 et $4 \%$ d'ammoniac, une augmentation plus faible (maximum de 10 points) que celles obtenues dans cette étude a été observée (Brown et al, 1987). De façon générale, nos résultats rentrent dans la gamme de ceux obtenus par Zoungrana (1994) sur les mêmes fourrages. L'augmentation des quantités de paille ingérées et l'amélioration de la dMO après traitement à l'urée peuvent s'expliquer par les modifications chimiques liées à la rupture des liaisons lignine-polyosides qui entrâ̂nent un ramollissement du fourrage mais également une augmentation des possibilités d'attaque des bactéries et des enzymes hydrolytiques et en conséquence la libération de nutriments digestibles (Spencer et Akin, 1980 ; Buettner et al, 1982).

Si l'on accepte l'hypothèse généralement admise selon laquelle l'aliment concentré ajouté à la ration garde ses caractéristiques propres, il est possible de calculer l'effet de la complémentation sur la valeur nutritive du fourrage associé. Avec Pennisetum pedicellatum complémenté avec $24 \%$ de tourteau de coton, la part revenant au fourrage dans la ration est de $61,5 \mathrm{~g} / \mathrm{kgP}^{0,75}$ avec une dMO de $58,7 \%$ et une quantité de matière organique digestible ingérée (MODi) de $36,1 \mathrm{gMS} / \mathrm{kgP}^{0,75}$. Le fourrage traité, distribué seul a la même $\operatorname{dMO}(58,4 \%)$ mais n'est ingéré qu'à $53,4 \mathrm{~g} / \mathrm{kgP}^{0,75}$ soit une MODi de $31,2 \mathrm{~g} / \mathrm{kgP}^{0,75}$. Dans ce cas, la complémentation est légèrement plus efficace que le traitement. Or, Pennisetum pedicellatum Trin non traité distribué seul ne peut pas assurer les besoins d'entretien des animaux. La complémentation ou le traitement chimique en améliore nettement la valeur nutritive. Pour ce type de fourrage, ces deux techniques peuvent être envisagées selon les moyens à la disposition du producteur.

Avec Schoenefeldia gracilis complémenté avec $13,4 \%$ de tourteau de coton, la part revenant au fourrage dans la ration est de $54,5 \mathrm{~g} / \mathrm{kgP}^{0,75}$ avec une dMO de $48,2 \%$ (MODi : 26,3 g/kgP 0.75 ). Ces valeurs sont inférieures à celles trouvées après traitement (respectivement $67,2 \mathrm{~g} / \mathrm{kgP}^{0,75}$ et $61,7 \%$ soit une MODi de 41,5 g/kgP ${ }^{0,75}$ ). La complémentation a permis une légère amélioration des caractéristiques nutritives du produit non traité, mais est moins efficace que le traitement chimique. Une augmentation de 25 à $30 \%$ ou $40 \%$ de la quantité de paille ingérée a été observée avec respectivement une complémentation à base de blocs 
« mélasse, urée, minéraux et vitamines » (Sansoucy et al, 1988), ou avec $180 \mathrm{~g}$ de mélasse urée (Nyarko-Dadohu et al, 1994). Tous ces résultats s'expliquent par une stimulation des activités de la flore ruminale consécutivement à l'apport de nutriments énergétiques et azotés. En effet, une teneur minimale de $7 \%$ de MAT nécessaire pour couvrir les besoins azotés des microorganismes du rumen responsables de l'activité cellulolytique et donc de l'ingestion est juste atteinte $(8 \%)$ avec la ration à base de $\mathrm{Sg}$, alors qu'elle était largement dépassée avec celle à base de $\mathrm{Pp}(13 \%)$. Cette dernière ration est donc plus favorable à l'activité microbienne.

Les résultats obtenus avec $\mathrm{Pp}$ et $\mathrm{Sg}$ peuvent s'expliquer par la différence de caractéristiques entre les deux fourrages ; Pp est une graminée à tiges plus robustes que celles de Sg. Elle peut déterminer une différence de réactivité au traitement alcalin. Ceci a d'ailleurs déterminé le niveau du tourteau de coton : $24 \%$ avec Pp contre $13 \%$ avec Sg. Sg complémenté apporte moins d'azote et d'énergie que $\mathrm{Pp}$ complémenté, ce qui peut être à l'origine de l'effet plus marqué de la complémentation avec cette dernière ration.

Les quantités de fourrage ingérées dépendent aussi de la quantité de fourrage mis à la disposition des animaux et donc de leur possibilité de trier (Haggar et Hamed, 1970 ; Zimmelink et al, 1972). Schoenefeldia gracilis, récolté précocement, n'est ingéré qu'à raison de $28,1 \mathrm{~g} / \mathrm{kgP}^{0,75}$ lorsque le pourcentage de refus est limité à $20 \%$ des quantités distribuées (essai 2). En revanche, les animaux en ingèrent $34,7 \mathrm{~g} / \mathrm{kgP}^{0,75}$ lorsque le pourcentage de refus est porté à $40 \%$ (essai 3). Ces valeurs restent inférieures aux résultats obtenus avec Pennisetum pedicellatum Trin, $50,9 \mathrm{~g} / \mathrm{kgP}^{0,75}$, et s'expliquent par la présence des inflorescences, facteur d'inappétence pour les animaux, puisque les refus en étaient essentiellement constitués. Le traitement de conservation à la dose de $2 \%$ d'urée améliore les quantités ingérées (+ $39 \%$ par rapport au fourrage non traité). Cela montre tout l'intérêt de réaliser des récoltes précoces de fourrage en période humide pour éviter chez Schoenefeldia gracilis la présence des inflorescences. Les graminées récoltées à maturité complète ne couvrent pas les besoins d'entretien des animaux, en raison des faibles teneurs en MAT et des faibles quantités de MOD ingérées (Chenost, 1975 ; Zoungrana, 1994).

\section{CONCLUSION}

Les traitements à l'urée de Pennisetum pedicellatum Trin et Schoenefeldia gracilis ont permis d'améliorer considérablement leurs quantités ingérées et la digestibilité de leurs composants chimiques. La couverture des besoins azotés des animaux alimentés avec ces fourrages a été assurée. La valeur nutritive ainsi obtenue est comparable à celle des végétaux exploités à un stade plus précoce comme le début épiaison pour Pennisetum pedicellatum Trin. De même, la complémentation avec le tourteau de coton a permis d'améliorer les ingestibilités et les digestibilités. Ces résultats permettent d'envisager deux alternatives à l'exploitation des fourrages étudiés :

- la récolte précoce au stade début épiaison pour Pp et avant apparition des inflorescences pour $\mathrm{Sg}$ avec ou sans traitement de conservation à $2 \%$ d'urée ;

- la récolte à l'état de paille, avec un traitement à $6 \%$ d'urée et à $40 \%$ d'humidité.

Le traitement des fourrages nécessite cependant la construction d'un silo, l'achat d'urée et d'accessoires (film en polyéthylène ou matériaux locaux pour assurer l'étanchéité).

La récolte des fourrages à un stade précoce, vers la fin de la période pluvieuse, représente une innovation au Burkina Faso. $\mathrm{Sa}$ vulgarisation devra être conduite avec 
méthode et efficacité car les conditions de mise en œuvre sont délicates et modifient les habitudes des éleveurs. À cette époque de l'année, les travaux agricoles ne sont pas terminés et les disponibilités en maind'œuvre pour réussir cette opération devront être mobilisées.

La complémentation reste une possibilité d'améliorer l'utilisation des fourrages tardifs, mais son utilisation est liée à la disponibilité en aliments riches en azote et en énergie. Depuis la dévaluation du franc CFA, les sous-produits agro-industriels sont de plus en plus exportés vers les pays industrialisés (Europe), et sont moins disponibles. Ces propositions pourraient être généralisées à de nombreux fourrages tropicaux.

\section{REMERCIEMENTS}

Les auteurs tiennent à remercier $\mathrm{S}$ Ouédraogo, observateur à l'Inera, pour sa contribution à la collecte des données, et $\mathrm{H}$ Zouré, chef de la cellule informatique à l'Inera, pour sa participation au traitement statistique des données.

\section{RÉFÉRENCES}

AOAC (1984) Official methods of analysis ( $13^{e}$ edn). Association of Official and Analytical Chemists, Washington DC, $114 \mathrm{p}$

Bougouma-Yaméogo V (1995) Valorisation des fourrages naturels récoltés au Burkina Faso (zones sahélienne et soudanienne). Traitement à l'urée de la biomasse et utilisation par les ruminants. Thèse, Ensa.M, Montpellier, $134 \mathrm{p}$

Bougouma-Yaméogo V, Cordesse R, Nianogo AJ, Inesta M, Nassa S (1996) Modifications chimiques et estimation de la dégradabilité de la matière sèche de quatre fourrages tropicaux traités à l'ammoniac ou à l'urée. Rev Elev Méd Vét Pays Trop 147, $689-700$

Brown WF(1988) Maturity and ammoniation effects on the feeding value of tropical grass hay. J Anim Sci $66,2224-2232$

Brown WF, Phillips DJ, Jones DB (1987) Ammoniation or cane molasses supplementation of low quality forage. J Anim Sci 64, 1205-1214

Buettner MRV, Lechtenberg VL, Hendrix S, Hertel JM (1982) Composition and digestion of ammoniated tall fescue (Festuca arundinacea S) hay. J Anim Sci 54,173

Chenost M (1975) La valeur alimentaire de Pangola (Digitaria decumbens Stent) et ses facteurs de variation, en zone tropicale humide. Ann Zootech 24, 327-349

Chenost M, Royer V, Centres JM, Gaillard F, Davis J (1993) Traitement des tiges de maïs à l'urée et utilisation pour la production laitière en région productrice de café et de banane en Tanzanie. Rev Elev Med Vét Pays Trop 46, 597-608

Guerin H, Richard D, Friot D, Sall S (1990) Amélioration de la performance des ruminants dans les pays en voie de développement avec référence spéciale aux aspects nutritionels. $41^{\mathrm{e}}$ réunion de la Fédération européenne de zootechnie, 9-12 Juillet Toulouse, 180-181

Haggar RJ, Ahmed MB (1970) Scasonal production of Andropogon gayanus. II- Seasonal changes in digestibility and feed intake. J Agric Sci 75 , 369-373

Hassoun P (1987) Amélioration de la valeur nutritive de la bagasse de canne à sucre par un traitement à l'ammoniac (genéré par hydrolyse de l'urée) et son utilisation par les ruminants. Thèse doctorat, USTL, Montpellier, $225 \mathrm{p}$

Nefzaoui A (1994) Adaptation de l'utilisation et du traitement des pailles aux conditions d'Afrique du Nord. In : Les pailles dans l'alimentation des ruminants en zone méditerranéenne (Tisserand JL,ed), Zaragoza : IAMZ, Ciheam (série B : Études et Recherches $n^{\circ} 6$ ), 61-78

Nyarko-Badohu DK, Kayouli C, Ba AA, Gasmi A (1994) Valorisation des pailles de céréales en alimentation des ovins dans le nord de la Tunisie : traitement à l'urée et à l'ammoniac et complémentation par des blocs mélasse-urée. In : Les pailles dans l'alimentation des ruminants en zone méditerranéenne (Tisserand JL, ed), Zaragoza : IAMZ, CIHEAM (série B : Études et Recherches n ${ }^{\circ} 6$ ) $129-142$

Robertson JB, Van Soest PJ (1981) The detergent system of analysis and its application to human foods. In : The Analysis of Dietary Fiber (James WPT, Theander O, eds), Marcell Dekker, New York, $123 \mathrm{p}$

Sansoucy R, Gys Aarts, Preston TR (1988) Molasseurea blocks as a multinutrient-supplement for ruminants. FAO, Animal Production and Health, Paper $72,263-278$

Sauvant D (1981) Prévision de la valeur énergétique des aliments concentré et composés pour les ruminants. In : Prévision de la valeur nutritive des aliments des ruminants. Tables de prévision de la valeur alimentaire des fourrages (Andrieu J, Demarquilly C, Wegat-Litre, eds), Inra Publication, Paris, 237-257

Sas (1982) SAS User's Guide : statistics. SAS Inst Inc, Carry, NC 40 p 
Schiere JB, Nell AJ, Ibrahim MNM (1988) Alimentation des animaux avec de la paille de riz traitée à l'urée et à l'ammoniac. Rev Mond Zootech 65. $31-42$

Spencer RR, Akim DE (1980) Rumen microbial degradation of potassium hydroxide treated coastal bermudograss leaf blades examined by election microscopy. J Anim Sci 51, 1189
Zimmelink G, Haggar RJ, Davies JH (1972) A note on the volontary intake of Andropongon gavanus hay by cattle as affecting by level of feeding. Anim Prod 15, 85-88

Zoungrana C (1994) Composition chimique et valeur nutritive des herbacées et des ligneux des pâturages soudaniens et des sous-produits du Burkina Faso. Thèse d'État, université de Ouagadougou, Burkina Faso, $209 \mathrm{p}$ 